\title{
Magnetic field enhanced coherence length in cold atomic gases
}

\author{
O. Sigwarth, ${ }^{1, \text { * }}$ G. Labeyrie, ${ }^{2}$ T. Jonckheere,,${ }^{1, \text { 由 }}$ D. Delande, ${ }^{1}$ R. Kaiser, ${ }^{2}$ and C. Miniatura ${ }^{2}$ \\ ${ }^{1}$ Laboratoire Kastler Brossel, Tour 12, Etage 1, 4 Place Jussieu, F-75005 Paris, France \\ ${ }^{2}$ Institut Non Linéaire de Nice, 1361 route des Lucioles, F-06560 Valbonne, France.
}

(Dated: November 15, 2018)

\begin{abstract}
We study the effect of an external magnetic field on coherent backscattering (CBS) of light from a cold rubidium vapor. We observe that the CBS enhancement factor can be increased with $B$. This surprising behavior shows that the coherence length of the system can be increased by adding a magnetic field, in sharp contrast with usual situations. This is mainly due to the lifting of the degeneracy between Zeeman sublevels. We find good agreement between our experimental data and a full Monte-Carlo simulation, taking into account the magneto-optical effects and the geometry of the atomic cloud.
\end{abstract}

PACS numbers: 42.25.Dd, 33.55.Ad, 32.80.Pj

During the last few years, ultracold atomic gases have become an ideal experimental and theoretical tool to investigate quantum phase transitions. Well-known examples are the Bose-Einstein condensation, degenerate Fermi gases and the Mott-Hubbard metal-insulator transition [1]. Cold atomic gases also constitute extremely interesting samples to study weak and strong localization of waves in disordered media. Indeed, contrary to typical condensed matter samples (e.g. multiple scattering of electrons in metals or semiconductors), atoms interact very resonantly with light. Thus the scattering processes at work can be more finely controlled and analyzed, opening the way to new studies in localization phenomena. Of special interest is the so-called mesoscopic regime, where interefences between multiply scattered waves are not washed out by the disorder. Here the notion of coherence length $L_{\phi}$ is crucial since it sets the characteristic length scale at which interference effects play a role in transport. For sample sizes $L<L_{\phi}$, these interference effects give rise to the so-called weak localization corrections of transport [2]. They originate from the interference between waves contrapropagating along loops in a scattering path. These interferences survive the configuration average and generally induce a negative correction to the usual classical Drude-Boltzmann conductance, as evidenced in 2D-electron gases [3]. In optics, a related phenomenon is the coherent backscattering (CBS) effect [4]. Here the diffuse reflexion off the sample is enhanced around backscattering in a narrow angular range $\propto 1 /(k \ell) \ll 1$ ( $k$ is the light wavevector and $\ell$ the scattering mean free path) because of constructive interferences between waves propagating along reversed paths. This interference effect is similar to the previous closed-loops interference and is maximum when the complex amplitudes of the reversed paths are exactly balanced. This is the case for time-reversal symmetric systems where, for a convenient choice of polarization channel, an enhancement factor close to 2 is observed [4].

These interferential corrections to transport are particularly sensitive to any physical source of dephasing susceptible to suppress interference. Some examples are inelastic scattering (electron-phonon interaction [2], saturation of an atomic transition [5]), spin-flip scattering [2] or internal degeneracies [6]. In this respect, the use of a magnetic field, which breaks time-reversal invariance, offers an efficient tool to investigate phase coherent effects 3]. In an electron gas, the loops of a diffusion path now enclose a magnetic flux which alters interference effects. As a consequence the coherence length is reduced as $B$ is increased. These modifications of the weak localization corrections have been experimentally verified on magnetoconductance measurements [3]. In optics, one gets the same kind of results but evidenced in CBS studies. Adding an external magnetic field breaks the time-reversal symmetry, leading to unbalanced interfering amplitudes and consequently to a decrease of the enhancement factor [7, 8]. This is so because, by modifying the refractive index of the medium, the magnetic field induces a Faraday effect which alters the phase difference at propagation and, in turn, gradually destroys the CBS effect.

In this letter, we report the first study of the CBS enhancement factor with cold atoms subjected to an external magnetic field $B$. We show an a priori surprising restoration of interference effects. Correspondingly, the coherence length is increased. This rather paradoxical result stems from the existence of an internal structure of the atoms (a non-zero angular momentum, hence degenerate, ground state) which is already responsible, at zero field, for unbalanced interfering amplitudes and reduced interference contrast [6, 9, 10. The magnetic field breaks the degeneracy of the atomic ground state and splits the atomic resonance line (Zeeman effect). As $B$ is increased, less and less Zeeman levels participate in the scattering process. At large enough fields, atoms behave as effective non-degenerate two-level systems and full multiple scattering interference contrast is restored.

The paper is organized as follows. We first briefly describe the experiment and our observations. We then discuss the basic physical ingredients and the principle 


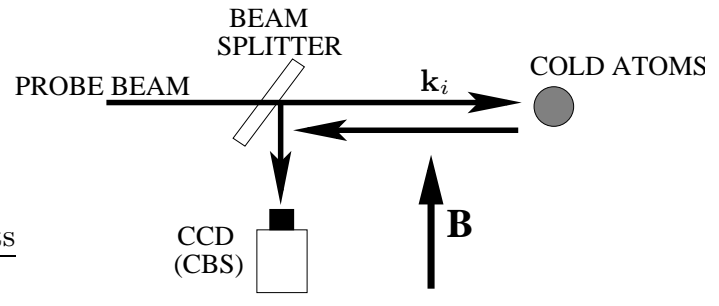

FIG. 1: Principle of the experiment. An external magnetic field $\mathbf{B}$ is applied orthogonally to the incident wavevector $\mathbf{k}_{i}$. A circularly-polarized laser beam is shined at the cold atomic cloud, and the backscattered far-field intensity distribution is recorded on a CCD (detection in the parallel helicity channel).

of the Monte-Carlo simulation. Then, we identify the various mechanisms at work, trying to give some simple physical pictures.

Our CBS experimental setup and its typical relevant parameters have been described in [6]. We have here added a pair of Helmholtz coils to produce a uniform external magnetic field $\mathbf{B}$ perpendicular to the incoming light wavevector $\mathbf{k}_{i}$ (Fig (1). The CBS probe is a weakly saturating laser beam (linewidth less than $2 \mathrm{MHz}$ ) with initial circular polarization $\boldsymbol{\epsilon}_{\boldsymbol{i}}$, shined at the atomic cloud while the magneto-optical trap (MOT) is off. It is quasiresonant with the closed $F=3 \rightarrow F^{\prime}=4$ transition of the $\mathrm{D} 2$ line of $\mathrm{Rb}^{85}$ (wavelength $\lambda=780 \mathrm{~nm}$, linewidth $\Gamma / 2 \pi=5.9 \mathrm{MHz}$ ). The magnetic field is switched on slightly before the CBS probe is applied and the optical thickness at zero field is $b=31$. During the measurements, for each value of $B$, the CBS laser is maintained at resonance with the Zeeman shifted $m_{F}=3 \rightarrow m_{F^{\prime}}=4$ transition (quantization axis along $\mathbf{B}$ ). This is an essential feature to understand our results and the interpretation given below. The CBS signal is recorded in the so-called parallel helicity channel, where the detected helicity is identical to the incident one. Results are shown in Fig 2 where the CBS enhancement factor $\alpha$ (peak to background ratio) is plotted as a function of $B$. It is observed that $\alpha$, initially very small $(\approx 1.05$ for $B=0)$, sharply increases with $B$ and saturates around 1.33 above $30 \mathrm{G}$. This behavior is obviously very different from the classical case, where $\alpha$ is reduced by the presence of $B$ in all reported instances [8].

In the weak localization regime 11, a multiple scattering path is depicted as a succession of single scattering events (described by their scattering amplitudes) separated by average propagation in the effective medium (described by the refractive optical index). When a magnetic field $\mathbf{B}$ is applied, the atomic energy levels are shifted by an amount $\Delta E=g m \mu B \hbar$, where $g$ is the Landé factor (respectively $1 / 3$ and $1 / 2$ for the ground and excited state considered here), $m$ the magnetic quantum number and $\mu / 2 \pi=1.4 \mathrm{MHz} / \mathrm{G}$ (Bohr magneton). The Zeeman splitting into a non-degenerate structure af-

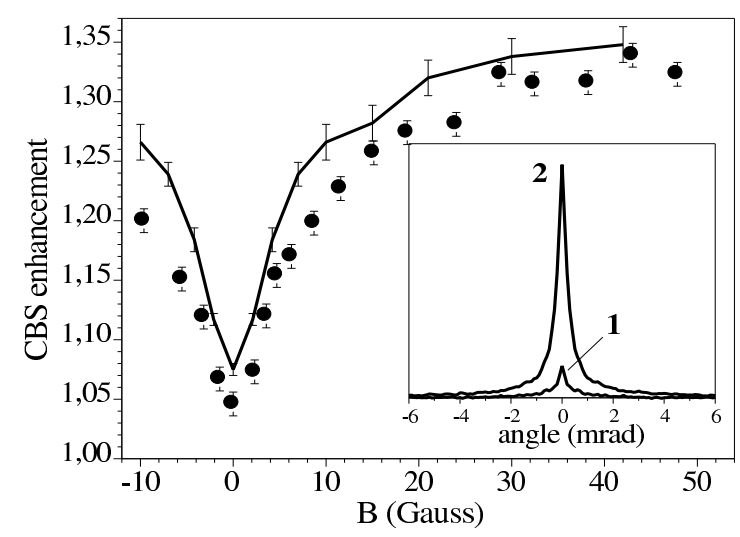

FIG. 2: CBS enhancement factor of light on a cold Rb atomic cloud, measured in the parallel helicity channel $h \| h$, as a function of the transverse magnetic field $B$ (circles); it shows a dramatic increase at non-zero magnetic field whereas timereversal symmetry is broken. For each $B$ value, the laser is tuned to resonance with the $m_{F}=3 \rightarrow m_{F^{\prime}}=4$ transition. The solid line is the result of the Monte-Carlo simulation (no adjustable parameter). The inset shows two (angularly averaged) experimental CBS peak profiles for $B=0(1)$ and $B=43$ G (2).

fects both the properties of an individual scatterer and of the effective medium [12]. An essential difference between usual scatterers and atoms is that atoms have an extremely resonant scattering cross-section (quality factor $\simeq 10^{8}$ ). Hence few Gauss are enough to induce a Zeeman shift comparable to the resonance linewidth $\Gamma$, and consequently a large modification of the scattering cross-section. This is also why the CBS enhancement factor displays dramatic changes, even at moderate $B$, in sharp contrast with published experimental results on standard scatterers which required magnetic fields in the Tesla range [8]. An additional complication with atoms is the existence of several Zeeman sublevels. The scattering process can induce a transition between different Zeeman sublevels (inelastic Raman scattering).

The scattering amplitude of an incident photon $|\mathbf{k} \boldsymbol{\epsilon}\rangle$ onto the outgoing light state $\left|\mathbf{k}^{\prime} \boldsymbol{\epsilon}^{\prime}\right\rangle$ by an atom whose initial state $\left|F m_{1}\right\rangle$ is changed to $\left|F m_{2}\right\rangle$ is described by the scattering tensor $T_{m_{1}, m_{2}}$ :

$$
\overline{\boldsymbol{\epsilon}^{\prime}} \cdot T_{m_{1}, m_{2}} \cdot \boldsymbol{\epsilon}=\sum_{m^{\prime}=-F^{\prime}}^{F^{\prime}} \frac{\left\langle F m_{2}\left|\mathbf{d} \cdot \overline{\boldsymbol{\epsilon}^{\prime}}\right| F^{\prime} m^{\prime}\right\rangle\left\langle F^{\prime} m^{\prime}|\mathbf{d} . \boldsymbol{\epsilon}| F m_{1}\right\rangle}{\delta+\mu B\left(g m_{1}-g^{\prime} m^{\prime}\right)+i \Gamma / 2}
$$

where $\mathbf{d}$ is the atomic dipole operator and $\delta=\omega-\omega_{0}$ is the detuning of the laser frequency $\omega$ from the zerofield atomic frequency $\omega_{0}$. The scattered photon has a frequency $\omega^{\prime}=\omega+g \mu B\left(m_{1}-m_{2}\right)$ and polarization $\Delta_{\mathbf{k}^{\prime}} T_{m_{1}, m_{2}} \boldsymbol{\epsilon}$, where $\Delta_{\mathbf{k}^{\prime}}$ is the projector on the plane perpendicular to $\mathbf{k}^{\prime}$. In eq. (11), the denominator is sim- 
ply the detuning of the incoming laser from the (Zeeman shifted) $\left|F m_{1}\right\rangle \rightarrow\left|F^{\prime} m^{\prime}\right\rangle$ transition.

In the presence of a magnetic field, the effective medium displays well documented magneto-optical anisotropies [7, 13]: a polarization propagating along a given direction $\hat{\mathbf{r}}$ decomposes on two eigenmodes which propagate with a differential phaseshift (Faraday effect) and a differential attenuation (Cotton-Mouton effect). When atoms are uniformly distributed over the Zeeman ground states (a good approximation for atoms produced in a MOT) and when optical pumping is negligible [14], the complex refractive index matrix reads:

$$
N(\omega, \hat{\mathbf{r}})=1-\frac{1}{2 k \ell_{0}} \frac{\Gamma / 2}{\delta+\mathrm{i} \Gamma / 2} \Delta_{\boldsymbol{r}}\left(\begin{array}{ccc}
\zeta & \eta & 0 \\
-\eta & \zeta & 0 \\
0 & 0 & \zeta+\xi
\end{array}\right) \Delta_{r}
$$

Here $\ell_{0}$ is the mean free path at zero field, $\Delta_{r}$ is the projector onto the plane transverse to $\hat{\mathbf{r}}$ and $\zeta, \eta$ and $\xi$ are simple functions of $\phi=\frac{i \mu B}{\delta+i \Gamma / 2}(\zeta$ and $\xi$ are even in $B$ whereas $\eta$ is odd). Their detailed expressions will be published elsewhere. $\zeta$ describes the normal extinction of the light in the medium, while $\eta$ and $\xi$ respectively describe the Faraday rotation and the Cotton-Mouton effect.

The scattering (1) and the index (2) matrices are the basic ingredients used in a Monte-Carlo simulation [9]. The enhancement factor at exact backscattering is obtained as $\alpha=1+I_{\text {coh }} /\left(I_{\text {single }}+I_{\text {inc }}\right)$ where $I_{\text {single }}$ is the single scattering contribution, $I_{\text {inc }}$ the diffuse contribution (incoherent intensity) and $I_{\text {coh }}$ the multiple scattering interference contribution (coherent intensity). Our numerical accuracy limits calculations up to $B \approx 40 G$. Results are shown in Figs. 22 and 31 The calculated enhancement factor is rapidly increasing from $\alpha=1.07$ at $0 \mathrm{G}$ to $\alpha=1.35$ at $42 \mathrm{G}$, in good quantitative agreement with the experimental observation. This agreement proves that our approach catches the most important physical mechanisms at work. In order to identify these mechanisms, we plot in Fig. 3(a), the ratio of the coherent over incoherent signals as a function of $B$. At $B=0$, the coherent intensity is much smaller than the incoherent one, featuring the CBS scrambling due to the atomic internal structure [6]. Then $I_{\mathrm{coh}} / I_{\mathrm{inc}}$ increases with $B$, featuring the restoration of the interference. As can be seen in Fig. 3(b), this is mainly due to the strong decrease of the incoherent intensity (crosses) even if the coherent intensity (circles) displays a small increase at small $B$. This decrease is related to the decrease of the atomic cross section: the optical thickness is lowered and multiple scattering is reduced. On the contrary, the coherent intensity increases at small $B$, because the reduction of the atomic degeneracy restores the interference. This phenomenon competes with the reduction of multiple scattering: at large $B$, the coherent intensity decreases, but more slowly than the incoherent one. Note, however, that the CBS enhancement factor remains noticeably smaller than 2 , even at large $B$, due to single scattering. The relative importance of single scattering is due to our specific cloud geometry 9 .

At large magnetic field $\mu B \gg \Gamma$, the Zeeman structure is sufficiently split and the $m_{F}=3 \rightarrow m_{F^{\prime}}=4$ optical transition remains the only one to be excited since the incoming light has been set at resonance with it $(\delta=\mu B)$. $I_{\text {coh }} / I_{\text {inc }}$ then approaches 1 because one can envision this transition as being closed and isolated, yielding an effective two-level system with no internal degeneracy. As a consequence, only left-circular photons with respect to B can be absorbed and emitted at each scattering and full interference contrast is restored. Note that the single scattering contribution cannot be removed by polarization analysis without removing the multiple scattering contribution. We have also checked that the limit $I_{\text {coh }} / I_{\text {inc }} \rightarrow 1$ is achieved independently of the polarization channel and of the relative orientation of $\mathbf{B}$ and $\mathbf{k}_{i}$.

This restoration of the multiple scattering interference contrast can be associated with an increase of the coherence length $L_{\phi}$ as $B$ is increased. Indeed, the contributions of the various scattering orders $p$ can be extracted from the Monte-Carlo calculation. For each value of $B$, we found a roughly exponential decrease of the ratio $I_{\text {coh }}^{(p)} / I_{\text {inc }}^{(p)} \approx \exp \left(-p / p_{0}\right)$. The coherence length is simply $L_{\phi}=p_{0} \ell$ where $\ell$ is the average scattering mean free path calculated at $B$. As reported in Fig. [ $p_{0}$ increases roughly linearly with $B$. This is in sharp contrast with usual solid-state samples where $L_{\phi}$ is reduced because of the Faraday effect which is proportional to the path length and to the magnetic field. In our case, the Faraday effect can be shown to vanish at large $B$ while, at the same time, the destructive impact of the Zeeman degeneracy is removed, as previously discussed. The residual coherence loss is thus due to deviations from the two-level approximation, i.e. residual scattering amplitude due to atomic transitions involving other (detuned from resonance) Zeeman sublevels. From eq. (11), this amplitude scales as $1 / B$, yielding a coherence length proportional to $B$, in agreement with our numerical results. The mesoscopic regime is achieved when $L_{\phi} \approx L$, or equivalently when $p_{0} \approx b$. At large $B$, the optical thickness is $b \approx 5$, hence the mesoscopic criterion is fulfilled when $B \gtrsim 15 G$.

Using our Monte-Carlo calculation, we have also quantitatively studied the impact of magneto-optical effects at propagation (Eq2) on the enhancement factor. They also appear to be negligible for $\mu B<\Gamma(\mu B=\Gamma$ corresponds to $B=4.2 \mathrm{G}$ in our experiment). For intermediate $B$, they cause the enhancement factor to be slightly lowered. In any case, they play no crucial role in the increase of $\alpha$ and can be neglected in a qualitative approach. This situation is very different from the classical one, where Faraday rotation is the keystone to explain the height and shape of the CBS cone [8].

This means, in turn, that the physics at work here is 

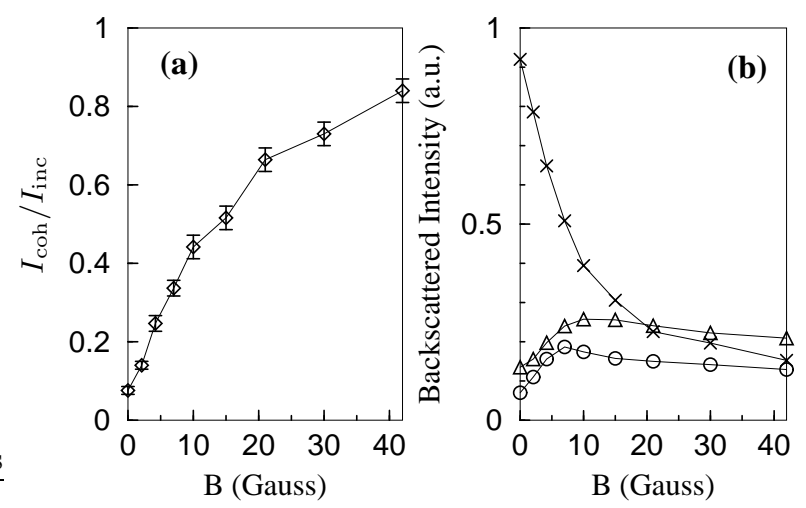

FIG. 3: Results of the Monte-Carlo simulations. (a) shows the increase of the ratio of coherent over incoherent intensity with $B$. (b) Contribution of single scattering (triangles), incoherent (crosses) and coherent (circles) multiple scattering to the total intensity in the backward direction. Lines are drawn to guide the eyes.

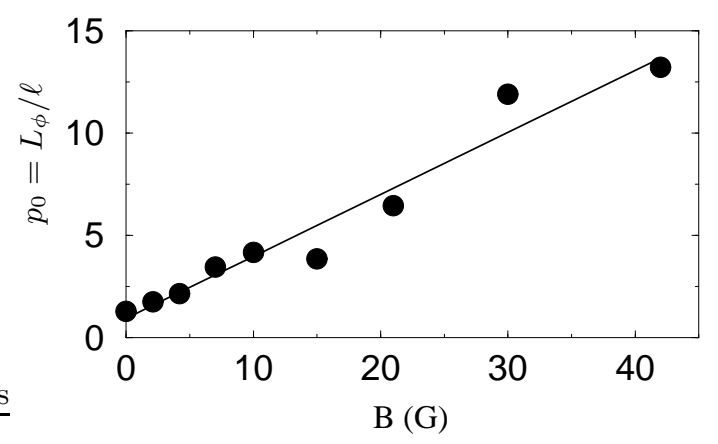

FIG. 4: Plot of $p_{0}=L_{\phi} / \ell$ versus $B$, as extracted from our Monte-Carlo calculation, as a function of the magnetic field $B$. In sharp contrast with the usual behaviour, $p_{0} i$ ncreases with $B$, roughly linearly (the solid line is drawn to guide the eye). This is due to the lifting of the Zeeman degeneracy. The points dispersion reflects the numerical accuracy.

the drastic modification of the scattering properties induced by the effective reduction of the internal structure. This effect has to be compared with the one observed in normal metal rings seeded with paramagnetic impurities [15]. There, at low fields, the Aharonov-Bohm (AB) oscillation amplitude is strongly reduced because of spin-flip scattering. This is equivalent to the observation of low CBS enhancement factors because of internal degeneracies. At high fields and low temperatures $\left(\mu B \gg k_{B} T\right)$, all impurities and electrons spins are aligned with $\mathbf{B}$. As in our experiment, $\mathbf{B}$ freezes the internal degrees of freedom of the scatterers. Electron spin fluctuations at scattering are then suppressed and full AB-oscillation amplitude is recovered.
In conclusion, we have observed a large increase of the CBS enhancement factor in the presence of a magnetic field, which splits the degenerate atomic transition into several Zeeman components. At high magnetic field, most of them are out of resonance, leaving an effective two-level system where the two interfering paths have almost equal amplitudes. Use of this magnetically-induced effective reduction of the atomic internal degeneracy may offer an interesting route in the quest of strong localization of light in cold atomic vapors.

We thank CNRS and the PACA Region for financial support. Laboratoire Kastler Brossel is laboratoire de l'Université Pierre et Marie Curie et de l'Ecole Normale Supérieure, UMR 8552 du CNRS.

* Electronic address: sigwarth@spectro.jussieu.fr

$\dagger$ Now at: Centre de Physique Théorique, Campus de Luminy, case 907, 13009 Marseille, France.

[1] M.H. Anderson et al., Science 269, 198 (1995); K.B. Davis et al., Phys. Rev. Lett. 75, 3969 (1995); B. DeMarco and D.S. Jin, Science 285, 1703 (1999); M. Greiner et al., Nature 415, 39 (2002).

[2] Mesoscopic Quantum Physics, E. Akkermans, G. Montambaux, J.-L. Pichard and J. Zinn-Justin, Eds., Elsevier Science B.V. (North Holland, Amsterdam, 1995).

[3] G. Bergmann, Phys. Rep. 107, 1 (1984).

[4] D. S. Wiersma, M. P. van Albada, B. A. van Tiggelen, A. Lagendijk, Phys. Rev. Lett. 74, 4193 (1995) ; Y. Bidel et al., Phys. Rev. Lett. 88, 203902 (2002).

[5] T. Chanelière et al., to be published in Phys. Rev. E (2004).

[6] G. Labeyrie et al., Phys. Rev. Lett. 83, 5266 (1999); T. Jonckheere et al., Phys. Rev. Lett. 85, 4269 (2000).

[7] A. S. Martinez and R. Maynard, Phys. Rev. B 50, 3714 (1994); B. A. van Tiggelen, R. Maynard, and T. M. Nieuwenhuizen, Phys. Rev. E 53, 2881 (1996).

[8] F. A. Erbacher, R. Lenke and G. Maret, Europhys. Lett. 21, 551 (1993); R. Lenke and G. Maret, Eur. Phys. J. B 17, 171 (2000); R. Lenke, R. Lehner and G. Maret, Europhys. Lett. 52, 620 (2000).

[9] G. Labeyrie et al., Phys. Rev. A 67, 033814 (2003); G. Labeyrie et al., Europhys. Lett. 61, 327 (2003).

[10] P. Kulatunga et al., Phys. Rev. A 68, 033816 (2003) ; D.V. Kupriyanov et al., Phys. Rev. A 69, 033801 (2004).

[11] A. Lagendijk and B. A. van Tiggelen, Phys. Rep. 270, 143 (1996).

[12] G. Labeyrie et al., Phys. Rev. Lett. 89, 163901 (2002).

[13] S. Franke-Arnold, M. Arndt, and A. Zeilinger, J. Phys. B.: At. Mol. Opt. Phys. 34, 2527 (2001); G. Labeyrie, C. Miniatura and R. Kaiser, Phys. Rev. A 64, 033402 (2001).

[14] This assumption is likely to be correct if the incident light is kept resonant with the $m_{F}=3 \rightarrow m_{F^{\prime}}=4$ transition while increasing $B$, like in our experiment.

[15] S. Washburn and R. Webb, Adv. Phys. 35, 375 (1986). 